79 Gerstein HC, Miller ME, Byington RP et al; ACCORD Study Group. Effects of intensive glucose lowering in type 2 diabetes. N Engl J Med 2008;358:2545-59.

80 Ismail-Beigi F, Craven T, Banerji MA et al. Effect of intensive treatment of hyperglycaemia on microvascular outcomes in type 2 diabetes: an analysis of the ACCORD randomised trial. Lancet 2010;376:419-30.

81 Hemmingsen B, Lund SS, Gluud C et al. Targeting intensive glycaemic control versus targeting conventional glycaemic control for type 2 diabetes mellitus. Cochrane Database Syst Rev 2013;11:CD008143.

82 Fullerton B, Jeitler K, Seitz M et al. Intensive glucose control versus conventional glucose control for type 1 diabetes mellitus. Cochrane Database Syst Rev 2014;2:CD009122.

83 Cushman WC, Evans GW, Byington RP et al; ACCORD Study Group. Effects of intensive blood-pressure control in type 2 diabetes mellitus. N Engl J Med 2010;362:1575-85.

84 Ginsberg HN, Elam MB, Lovato LC, Crouse JR 3rd et al; ACCORD Study Group. Effects of combination lipid therapy in type 2 diabetes mellitus. N Engl J Med 2010;362:1563-74.

85 Von Eynatten M, Emser A, Cooper M et al. Renal safety and outcomes with linagliptin: meta-analysis of individual data for 5466 patients with type 2 diabetes. J Am Soc Nephrol 2012;23(Suppl 1):218A.

86 Cherney DZ, Perkins BA, Soleymanlou N et al. Renal hemodynamic effect of sodium-glucose cotransporter 2 inhibition in ptients with type 1 diabetes mellitius. Circulation 2014;129:587-97.

87 Perico N, Antiga L, Caroli A et al. Sirolimus therapy to halt the progression of ADPKD. J Am Soc Nephrol 2010;21:1031.

88 Serra AL, Poster D, Kistler AD et al. Sirolimus and kidney growth in autosomal dominant polycystic kidney disease. $N$ Eng J Med 2010;363:820

89 Walz G, Budde K, Mannaa M et al. Everolimus in patients with autosomal dominat polycystic kidney disease. N Eng J Med 2010;363:830.

90 Torres VE, Chapman AB, Devuyst O et al. Tolvaptan in patients with autosomal dominant polycystic kidney disease. N Eng J Med 2012;367:2407.

Address for correspondence: Prof PT Murray, School of Medicine, University College Dublin, Health Sciences Centre, Dublin 4, Ireland.

Email: patrick.murray@ucd.ie

\title{
Faints, fits and funny turns for the physician
}

\author{
Author: Rohani Omar ${ }^{A}$
}

The diagnosis and management of the dizzy patient presents the physician with significant challenges. Dizziness and imbalance are common complaints among the general population, affecting around one-third of people over the age of 65 years, and can result from a range of causes spanning many medical disciplines. The ability to take a thorough, accurate history with a logical framework for formulating a differential diagnosis is essential given the many ways that symptoms of dizziness can present. An understanding of the key features of the vestibular examination, and consideration of other pathologies including neurological and cardiac, are important. This conference was held with the aim of demystifying the dizzy patient by providing physicians with a practical approach to the assessment and management of dizziness, imbalance and 'funny turns'.

KEYWORDS: Dizziness, imbalance, vestibular, vertigo, syncope, seizures

Author: ${ }^{\text {A }}$ specialist registrar in audiovestibular medicine, University College London Hospitals NHS Foundation Trust, London, UK

\section{Introduction}

There can be few physicians so dedicated to their art that they do not experience a slight decline in spirits when they learn that their patient's complaint is dizziness. ${ }^{1}$

Presentations of dizziness, imbalance, blackouts and 'funny turns' are common, yet many physicians struggle with the diagnosis and management of these symptoms. On 30 April 2015, a conference was organised by the Royal College of Physicians with the aim of providing a clinically focused, practical approach to the management of this challenging patient population. Key features of history taking, clinical examination and formulating a differential diagnosis of common conditions were covered. Case studies were used to illustrate different conditions and allow for audience participation in the form of anonymous voting using response pads to answer questions.

\section{Epidemiology and principles of management}

Vertigo of a vestibular cause is common, affecting more than $5 \%$ of adults per year. Around one-third of people over the age of 65 years living in the community report symptoms of 
dizziness. ${ }^{2}$ It has been shown that half of patients suffering with symptoms of dizziness experience some degree of handicap and report significant anxiety or avoidance behaviours. ${ }^{3}$ Delays in diagnosing treatable conditions such as benign paroxysmal positional vertigo (BPPV) have a significant cost impact on the NHS; many patients are subjected to repeat medical attendances and are seen by several specialists before diagnosis. The aetiology of dizziness and imbalance spans a range of disciplines, including neurology, cardiology, neurootology, haematology and endocrinology, and therefore a well organised integrated network between specialties is essential to efficiently diagnose and manage these conditions, and prevent over-investigation and inappropriate treatment. In the management of the dizzy patient, general internal medicine knowledge is essential.

The ability to maintain balance requires integration and modulation of visual, vestibular and proprioceptive inputs. One of the great difficulties lies in teasing out what specific symptoms patients are describing when they say they are dizzy. A 'history-based algorithm' was offered by Dr Peter West, providing a logical framework by which to hang ideas in formulating a differential diagnosis (Fig 1). Other aspects of the clinical assessment of a dizzy patient were discussed, including the examination of eye movements and how to interpret abnormalities. The typical features of peripheral vestibular nystagmus were explained, and the importance of education, reassurance and a clear explanation to patients of how to perform vestibular rehabilitation exercises in the management of vestibular neuritis was highlighted. Patients need to have some understanding of vestibular compensation, which occurs at the level of the brainstem, and that lack of head movement will prevent compensation from occurring. The prolonged use of vestibular suppressant drugs can also be detrimental to rehabilitation, and psychological factors need addressing to manage anxiety and avoidance behaviours.

Current updates on the management of acute vertigo, particularly in differentiating between vestibular neuritis, cerebellar stroke or migraine, were presented by Prof Adolfo Bronstein. Decisions regarding urgent magnetic resonance imaging in an acutely vertiginous patient need to be made on the basis of a sound clinical history and examination. The head impulse test is extremely important, as a negative test could suggest central pathology and an urgent scan would be indicated. Other 'red flags' for urgent scanning were discussed, including associated acute hearing loss, neurological symptoms or signs, and new-onset severe occipital headache. ${ }^{4}$

This was followed by a series of presentations covering the various conditions which can cause funny turns and dizzy spells, including BPPV, epilepsy, cardiac-related problems, Ménière's disease, vestibular migraine and less common vestibular disorders such as vestibular paroxysmia, multiple sclerosis and superior semicircular canal dehiscence. BPPV is one of the more common causes of episodic dizziness in the adult population, and a description of the typical symptoms of BPPV, the pathophysiology, diagnosis on Dix-Hallpike testing and treatment with different particle repositioning manoeuvres were discussed by Dr Peter West. Aside from posterior canal $\mathrm{BPPV}$, other rarer causes of position vertigo such as horizontal and anterior canal BPPV, cupulolithiasis and central causes of abnormal Dix-Hallpike testing were discussed.
Pre-syncope is a common cause of funny turns, and a presentation by Dr Nicholas Gall described the causes and management of syncope, as well as risk stratification in identifying high-risk cases with arrhythmias or structural cardiac anomalies. In general, diagnostic tests for syncope/presyncope have low yield and are expensive. The initial assessment of syncope need not be complicated and much of the diagnosis is made on history. The National Institute for Health and Care Excellence guidelines on transient loss of consciousness (T-LoC) $2010^{5}$ provide a framework for diagnosing uncomplicated faints, listing features which would prompt a referral for urgent cardiovascular assessment (eg abnormal echocardiogram, heart failure, T-LoC during exertion) or referral to a specialist for suspected epileptic seizure. Differentiating between epileptic seizures, vasovagal syncope and non-epileptic attack disorder can be challenging, and the general principles of diagnosing epilepsy were presented by Prof Simon Shorvon.

Ménière's disease and vestibular migraine, different diseases in terms of pathophysiology, can be difficult to distinguish particularly with 'grey areas' such as Ménière's with headache and migraine with asymmetric auditory symptoms. This subject was discussed by Dr Louisa Murdin, contrasting the features of Ménière's disease according to the AAO-HNS 1995 criteria $^{6}$ with vestibular migraine, ${ }^{7}$ and highlighting how a neuro-otological assessment can help. Specialist vestibular tests such as electronystagmography and vestibular evoked myogenic potentials can be useful in differentiating Ménière's disease, vestibular migraine and other less common disorders such as superior semicircular canal dehiscence syndrome.

\section{The elderly and adolescents}

Falls in the elderly are commonplace and debilitating and are often preventable. While many are due to syncope and cardiovascular causes, a significant number are attributable to multisensory balance disorders with vestibular dysfunction being an underdiagnosed but treatable cause of falls in older people. The fact that the elderly present in a different way from younger people was highlighted. The results of a project by the North Tyneside falls and syncope service, where fallers were identified early before presentation and offered preventive care, were presented by Dr Joanna Lawson. Through a scheme involving postal triage tools and targeted assessments in the community carried out by nurses, physiotherapists and physicians, the intervention succeeded in identifying $82 \%$ of respondents to the postal questionnaire to be at high risk of falling and requiring direct action.

At the other end of the spectrum, dizziness and balance disorders in the adolescent and young adult population require tailored input due to their specific physical, psychological, emotional and intellectual needs, as discussed by Dr Katherine Harrop-Griffiths. In delivering care to this population, physicians should take into account the language adolescents use to express themselves, the fact that parents in clinic may limit their expression or cause embarrassment when discussing their problems, as well as the problems with taking up care due to school holidays, examinations and leaving for university. The pathology in young people can also be different to the older adult population, as they are less likely to have degenerative disorders such as BPPV. 


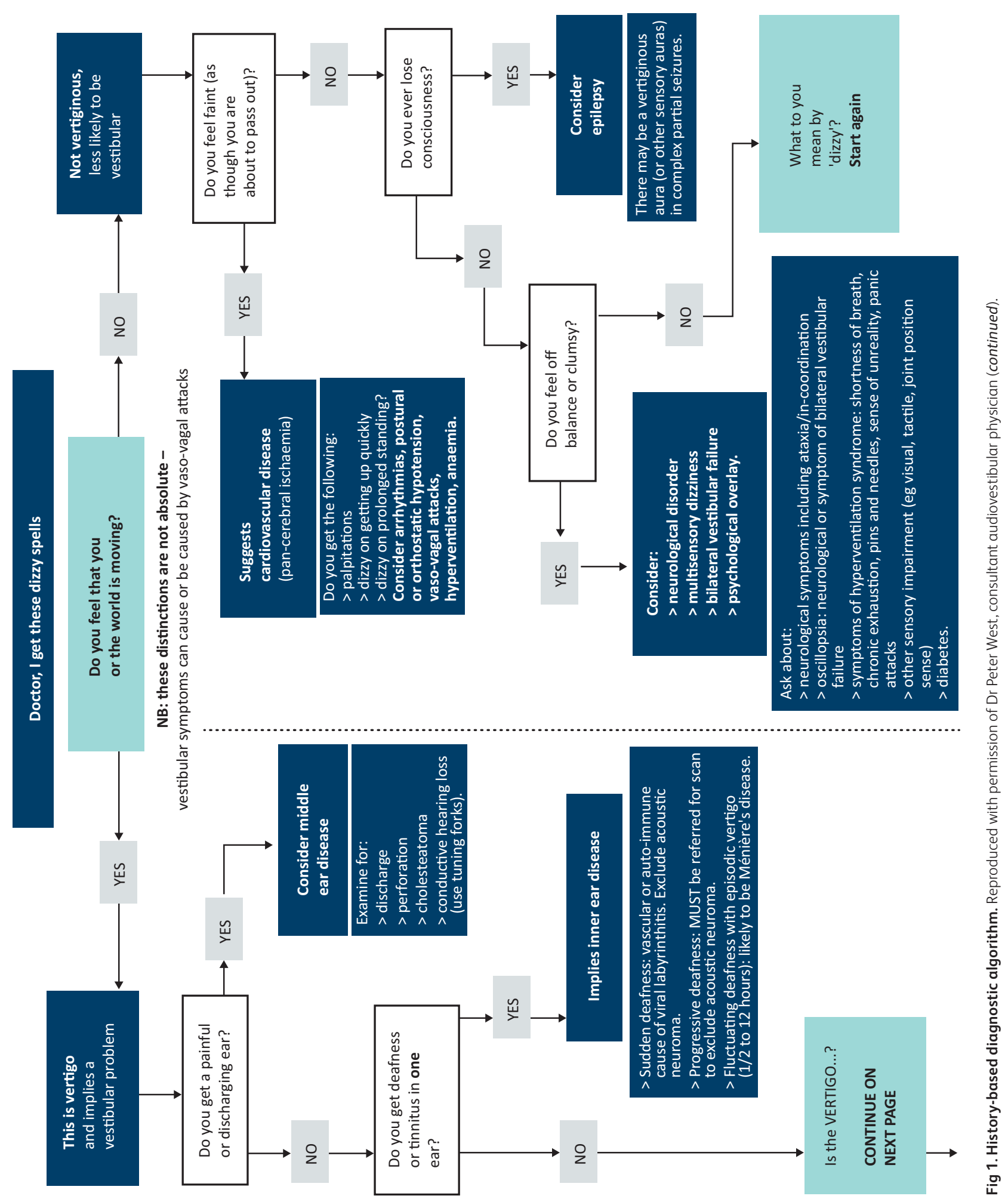




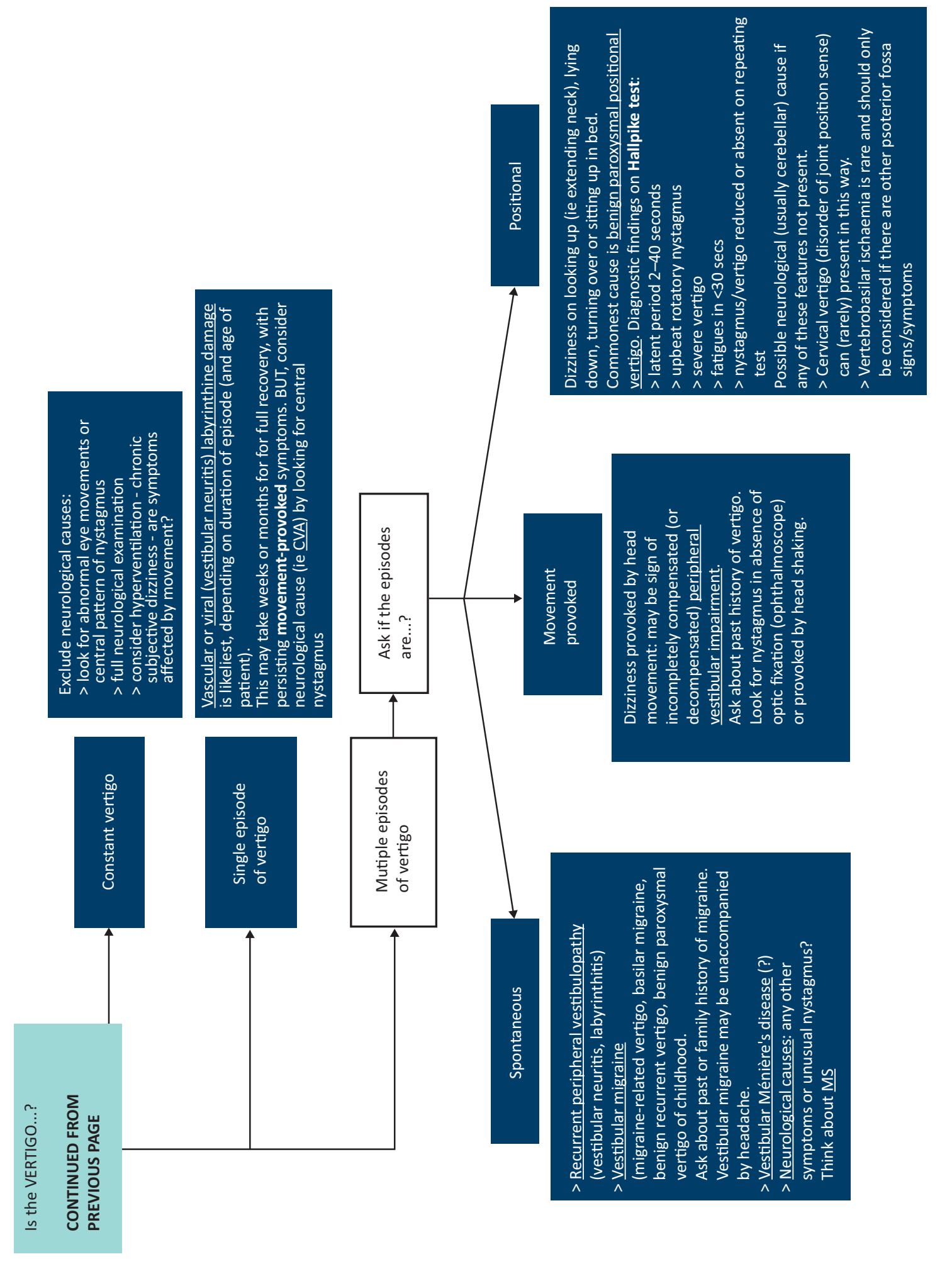




\section{A multidisciplinary approach}

Finally, physicians need to work closely with allied health professionals such as psychologists and physiotherapists in the multidisciplinary management of patients with dizziness, imbalance and falls. Psychologists play an important role in treating anxiety with the aim of eliminating avoidance behaviours that curb rehabilitation and lead to chronicity of dizziness and imbalance. In his presentation, Dr David Scott described treatment principles including breaking the cycle of safety behaviours, building tolerance and helping patients to 'approach not avoid' through the use of experiential methods. The role of vestibular physiotherapy in aiming to improve quality of life through exercise and education was discussed by Andrew Clements. Results from tests, such as dynamic posturography, are taken into account in creating targeted exercise programmes. The importance of educating patients and their carers on the process of central compensation in vestibular rehabilitation was highlighted.

To conclude, many conditions affecting a range of systems can result in dizziness and funny turns, hence the importance of good general internal medicine knowledge, a strategy for diagnosis, knowledge of appropriate investigative pathways and an integrated network between different specialties including rehabilitation support from psychologists and physiotherapists in order to ensure effective and efficient management.

\section{References}

1 Matthews WB. Practical neurology. Oxford: Blackwell, 1963.

2 Colledge NR, Wilson JA, Macintyre CC et al. The prevalence and characteristics of dizziness in an elderly community. Age Ageing 1994;23:117-20.

3 Yardley L, Redfern MS. Psychological factors influencing recovery from balance disorders. J Anxiety Disord 2001;15:107-19.

4 Seemungal BM, Bronstein AM. A practical approach to acute vertigo. Pract Neurol 2008;8:211-21.

5 National Institute for Health and Clinical Excellence. Transient loss of consciousness ('blackouts') management in adults and young people. London: NICE, 2010. Available online at www.nice.org.uk/ guidance/cg109/resources/guidance-transient-loss-of-consciousnessblackouts-management-in-adults-and-young-people-pdf [Accessed 30 September 2015].

6 Monsell EM. New and revised reporting guidelines from the Committee on Hearing and Equilibrium. American Academy of Otolaryngology-Head and Neck Surgery Foundation, Inc. Otolaryngol Head Neck Surg 1995;113:176-8.

7 Headache Classification Committee of the International Headache Society (IHS). The International Classification of Headache Disorders, 3rd edition (beta version). 2013. Available online at www.ihs-classification. org/_downloads/mixed/International-Headache-Classification-IIIICHD-III-2013-Beta.pdf [Accessed 30 September 2015].

Address for correspondence: Dr R Omar, Department of Audiology, St Helier Hospital, Wrythe Lane, Carshalton SM5 1AA, UK.

Email: rohaniomar@nhs.net 